\title{
PSO with Robust Disturbance Rejection Controller for Maneuvering Control the Altitude of UAV
}

\author{
WEI WANG \\ Xian Aeronautical University, Xi an, Shaanxi China.710077. \\ weiwang@xaau.edu.cn
}

\begin{abstract}
In this research article, particle swarm optimization (PSO) algorithm with variable weights and robust disturbance rejection (RDR) controller used to control the height of unmanned aerial vehicle (UAV). To solve the problem of controller parameters and robustness in obtaining a set of optimal solutions for the attitude control movements of the quadrotor UAV. The algorithm designed in this article is mainly composed of two parts; the first one in accordance with the iterative process, the distance of swarm particles with the variable weight of the dynamic change in the particle size of the global optimum set the coefficient to control its impact on the degree of inertial weight. The global optimal value of the result shows that the optimization algorithm is effective to adjust the parameters of the designed controller, which in result guarantee the quality in the control of the UAV and improve the design efficiency.
\end{abstract}

Keywords: UAV, Algorithm, Quadrotor.

\section{Introduction}

Unmanned aerial vehicles (UAVs), commonly known as drones, have found a wide range of applications during the past few years due to their low cost, efficiency and high mobility. UAVs have generally used in the military, deployed in remote and hostile areas where they perform their operations without endangering pilot lives. In the present era, these aircrafts are in high demand because they are much safer and more convenient than traditional aircrafts [1] and [2]. UAVs generally classified into two types based on their weight lifting capacity, engine type, range and maximum altitude etc. The first one fixed wing, these aircraft usually have high speed but they could only fly in continuous motion because of fixed mechanical structure. Second, one is the rotary wing aircraft such as UAVs; they are able to fly in any direction stand stationary in the air, able to hold on specific altitude in the air [3]. In reference [4] fuzzy PID controller and PID controller discussed, which plays an important role in achieving the stable attitude angle control of the aircraft, but there are some limitations that it has poor adaptability and can use for indoor flight operations. There is a huge demand of anti-interference control technology, where the aircraft requires high control accuracy and faces tremendous external interferences that provides stable and better flights 
results. Such as in reference [5], in order to minimize the air interferences and wind turbulences a self-immunity controller designed.

In reference [6] a cascade system of proportional derivative and active disturbance control proposed. In that controller the parameters of outer loop is control by PD controller and inner loop controlling done by ADRC. The use of PD control structure is easy and simple whereas Adaptive disturbance rejection controller (ADRC) has strong decoupling ability to minimize the internal and external interference. In reference [7] the theories of higher order sliding mode observer (HSMC) introduced in the ADRC which in result improved the ADRC, so that the controller has better control effects. Although there are several advantages of the ADRC like the accurate mathematical, model which is independent of the object, enhanced ability to estimate and compensate the uncertainties. However, there is no proper parameters for the tuning methods, using artificial error methods to make an efficient control system of the aircraft [8]. In reference [9] the parameters of adaptive disturbance rejection controller using particle swarm optimization PSO were discussed. On the other hand, there are some issues of local optimization and slow convergence in the standard particle swarm algorithm. By adjusting ADRC controller parameter of the quadrotor UAV using the variable weight hybrid particle swarm optimization algorithm the above problems can be resolved [10]. The whole article comprised of the following sections. Section I of the paper consists of an introduction, in section II the modelling of the UAV discussed. The adaptive disturbance rejection controller defined in section III of this paper. In section IV the improved particle swarm optimization algorithm is discussed. The complete article concluded in section V of this paper.

\section{Modelling of the UAV}

The dynamics of the quad-rotor aircraft used for the mathematical modelling and the kinematic equation of Euler angles of the UAV taken from [11] and [12];

$$
\begin{gathered}
\ddot{x}=\frac{(\cos \varphi \sin \theta \cos \psi+\sin \varphi \sin \psi) * u_{1}}{m} \\
\ddot{y}=\frac{(\cos \varphi \sin \theta \sin \psi+\sin \varphi \cos \psi) * u_{1}}{m} \\
\ddot{z}=-g+\frac{(\cos \varphi \cos \theta) * u_{1}}{m}-g \\
\ddot{\varphi}=\dot{\theta} \dot{\psi}\left(\frac{I_{y}-I_{z}}{I_{x}}\right)+\frac{1 u_{2}}{I_{x}} \\
\ddot{\theta}=\dot{\varphi} \dot{\psi}\left(\frac{I_{z}-I_{x}}{I_{y}}\right)+\frac{1 u_{3}}{I_{y}} \\
\ddot{\psi}=\dot{\varphi} \dot{\theta}\left(\frac{I_{x}-I_{y}}{I_{z}}\right)+\frac{1 u_{4}}{I_{z}}
\end{gathered}
$$

In equation (1) $x, y, z$ are the geographic coordinate system of the quadrotor UAV also known as Earth coordinates, $\emptyset, \theta, \psi$ are coordinate positions under reference coordinate system defined as roll angle, pitch angle and yaw angle. The $I_{x}, I_{y}, I_{z}$ are the three moments of inertia around $x, y, z$ axes, $g$ is the acceleration due to gravity of the aircraft, $\mathrm{m}$ is defined as the quality of the aircraft $\mathrm{u}_{1}, \mathrm{u}_{2}, \mathrm{u}_{3}, \mathrm{u}_{4}$ for the system control amount, the relationship with the quadrotor speed parameters as follows: 


$$
\begin{gathered}
u_{1}=k_{t}\left(\mu_{1}^{2}+\mu_{2}^{2}+\mu_{3}^{2}+\mu_{4}^{2}\right) \\
u_{2}=k_{t}\left(\mu_{1}^{2}-\mu_{3}^{2}\right) \\
u_{3}=k_{t}\left(\mu_{2}^{2}-\mu_{4}^{2}\right) \\
u_{4}=k_{d}\left(\mu_{1}^{2}-\mu_{2}^{2}+\mu_{3}^{2}-\mu_{4}^{2}\right)
\end{gathered}
$$

\section{Robust disturbance rejection controller}

The quadrotor robust disturbance controller define by the second order tracking differentiator TD, arrange the transition process, according to the set value $y_{d}$ arrange the transition process $y_{d 1}$ and extract the differential signal $y_{d 2}$.

$$
\left\{e=y_{d 1}-y_{d} y_{d 1}=y_{d 1}+h y_{d 2} y_{d 2}=y_{d 2}+h g s t\left(e, y_{d 2}, r, h_{0}\right)\right.
$$

In equation (3), $\mathrm{h}$ is the step, the $h_{0}$ and $\mathrm{h}$ are independent factors which has a filtering effect also called as the filter factor. The $r$ determines the tracking speed of the input signal differentiator, known as speed factor, gst function is the most rapid control function which is;

$$
u=\operatorname{gst}\left(y_{1}, y_{2}, r, h\right)
$$

According to controlled object, the expanded state observer ESO input $u_{1}$ and output y. The estimated state of controlled objects $Z_{1}, Z_{2}$ and the total amount of disturbance by the object $Z_{3}$.

$$
\begin{gathered}
\left\{e=Z_{1}-y \geq \operatorname{gal}\left(e, \alpha_{1}\right) g e_{1}=\operatorname{gal}\left(e, \alpha_{2}\right) Z_{1}=Z_{1}+h\left(Z_{2}-\gamma_{01} e\right) Z_{2}\right. \\
=Z_{2}+h\left(Z_{3}-\gamma_{02} \geq+b u\right) Z_{3}=Z_{3}+h\left(-g e_{1}\right)
\end{gathered}
$$

In equation (5) $\gamma_{01}, \gamma_{02}$ are greater than zero and require a proper adjustment of the parameter, $0<\alpha_{1}, \alpha_{2}<1$ usually take $\alpha_{1}=0.5, \alpha_{2}=0.25$, b is a constant, gal is the function of linear range width. The function of $g a l$ is defined as:

$$
\operatorname{gal}(e, \alpha)=\left\{|e|^{\alpha} \cdot \operatorname{sign}(e), 0\right.
$$

In equation (6) the value of $\alpha$ is taken as 0.5 . Nonlinear error feedback law, according to the input to in $e_{1}, e_{2}$ and $Z_{3}$ in order to determine the final amount of controlled object $u_{1}$.

$$
\left\{e_{1}=y_{d 1}-Z_{1} e_{2}=y_{d 2}-Z_{2} u_{10}=\gamma_{1} \operatorname{gal}\left(e_{1}, \alpha_{1}\right)+\gamma_{2} \operatorname{gal}\left(e_{2}, \alpha_{2}\right) u_{1}=u_{10}-\frac{Z_{3}}{b}\right.
$$

In equation (7) the gal function definition is same as defined in equation (6), but the value of $\alpha$ is different, in order to achieve good robustness and adaptability in the controller select the appropriate parameters $\gamma_{1}$ and $\gamma_{2}$.

\section{Particle swarm optimization}

The particle swarm optimization (PSO) is an optimization algorithm. Each particle of particle swarm optimization. It provides a possible set solution, which provides 
ultimate solution of the problems. By interacting with other particles, in order to find the optimal solution to the problem it constantly update itself with the status information of the entire population [13-15].

The particle group in $\mathrm{N}$ dimensional space consisting on $M$ particles, here $X_{i}=x_{i 1}, x_{i 2}$, $x_{i 3}, x_{i 4} \ldots . x_{i n} x$ represents the $i_{t h}$ position of a particle in the $n_{t h}$ dimensional space. $V_{i}=\left(v_{i 1}, v_{i 2}, v_{i 3} \ldots v_{i n}\right)$ here $v$ represents the $i_{t h}$ particle speed of $P_{g}=\left(p_{g 1}, p_{g 2}, p_{g 3}\right.$, $\left.p_{g 4} \ldots . . p_{g n}\right)$.

Each particle in PSO continuously update its velocity and position in space in an iterative process by its own population optimal values as shown in equation (9) and (10).

$$
\begin{gathered}
V_{k d}^{k+1}=\omega V_{i d}^{k}+c_{1} r_{1}\left(P_{i d}^{k}-X_{i d}^{k}\right)+c_{2} r_{2}\left(P_{g d}^{k}-X_{i d}^{k}\right) \\
X_{i d}^{k+1}=X_{i d}^{k}+V_{i d}^{k+1}
\end{gathered}
$$

In equation (9), (10) $\omega$ is the inertial weight; $d=1,2,3, \ldots \ldots n ; i=1,2,3, \ldots . m ; c_{1}, c_{2}$ is the acceleration factor, whose value is greater than zero. $r_{1}, r_{2}$ is a random number distributed in the interval of 0,$1 ; k$ is defined as number of current iterations.

The $\omega$ defines the inertial weights which reflects the velocity of the particles that has the same previous ability. $Y$ presents linear decrement inertia weights, as shown in equation (10), if the inertia weight value is large it favors global search, while the smaller inertia weight value is more conductive to local search.

In below equation the inertial weight $\omega$ used to define the velocity of the particle.

$$
\omega(k)=\frac{\omega_{\max }-k\left(\omega_{\max }-\omega_{\min }\right)}{T_{\max }}
$$

Where $\omega_{\max }$ and $\omega_{\min }$ for the maximum and minimum inertia weight value, $\mathrm{T}_{\max }$ is the maximum number of iterations.

\subsection{Improved basic flow of particle swarm algorithm}

1. In order to select maximum history and optimal particles set the position and velocity of all particles as per the fitness function value criteria.

2. Calculate the distance between each particle and the current global optimal position, get $L_{k i} \max , L_{k i}$ min from equation (8) update the inertia weight of each particle in next iteration $\omega$.

3. According to equation (8), (9), (10), update the position and velocity of each particle and calculate the fitness value.

4. The current value replaces the historical optimal value, if the current fitness value of the particle is better than its historical optimal value.

5. Replace the global optimal value, if the particle is better than the global historical optimal value. 
6. Determine whether the hybridization conditions that are continuous $H$ generation global optimal value remains unchanged, in the implementation of step 7.

7. Determine whether the termination condition is satisfied, the output is global optimal, otherwise step 2.

\subsection{Particle swarm algorithm RDR controller}

The mathematics of the quadrotor aircraft build in MATLAB/Simulink aircraft simulation model and design $R D R$ controller pair $U A V$ height and three-attitude angles to control. By combining $R D R$ controllers with improved particle swarm optimization (PSO), select the maximum impact on the performance of $R D R$ six parameters, using particle swarm optimization tuning, which ESO take $\gamma_{01}, \gamma_{02}, \gamma_{1}, \gamma_{2}$ and b such as shown in figure 1 below.

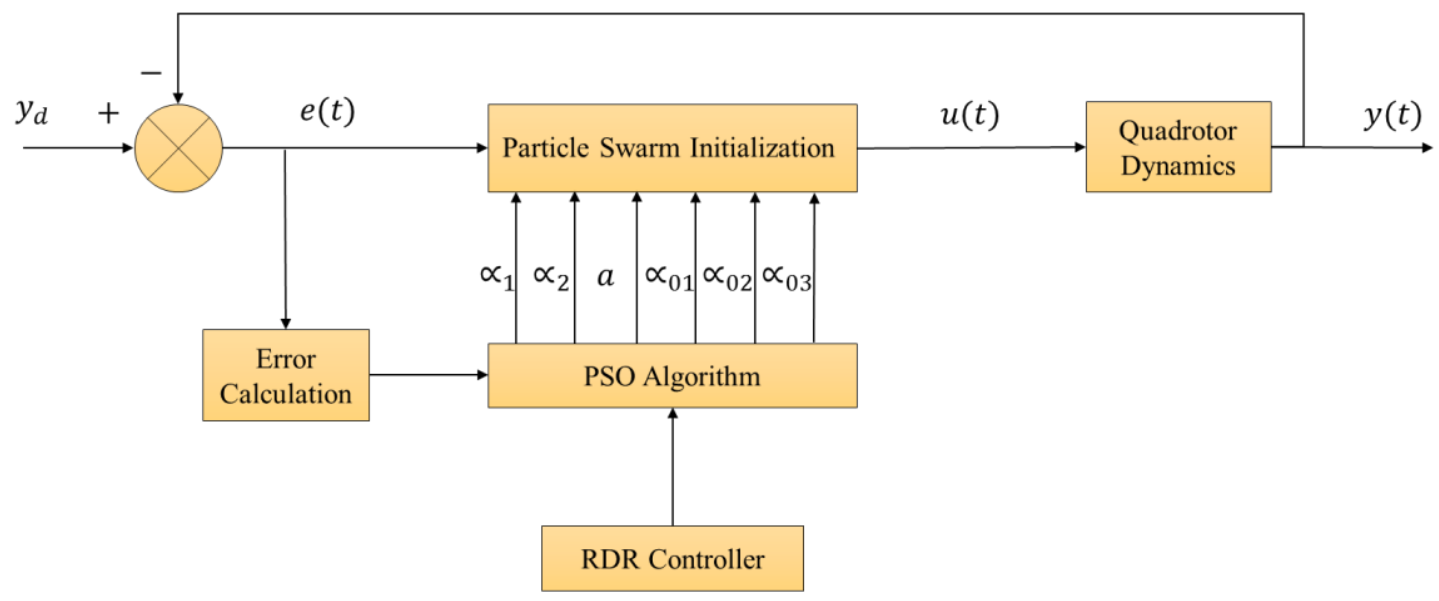

Figure: 1 Error performance indicators

\section{Simulation results analysis}

In this part of the manuscript, the robustness of the designed controller is defined. The parameters of quadrotor UAV is defined in table 1, and table 2 of this article contains PSO-RDR initialization parameters. The overall simulation model divided into two groups one is the altitude curve and the other one is no. of iterations for optimization. From figure 2, it shows the altitude curve of the UAV in which it can be clearly seen that in a 25 second time period, the combination of PSO-RDR perform better in achieving the reference height of 4.5 meters as compared to the traditional PSO controller. Although it can also observe from the simulated result that designed controller has better robustness and quick convergence speed.

Similarly, figure 3 shows that standard particle swarm optimization algorithm converges after 4 iterations, while the robust disturbance rejection based PSO algorithm converges after about 3 iterations this conclusion also shows the quick convergence response of the designed controller.

Table 1: Quadrotor Parameters 


\begin{tabular}{|c|c|c|}
\hline Parameters & Values & Units \\
\hline$m$ & 0.888 & $\mathrm{~kg}$ \\
\hline$l$ & 0.2335 & $\mathrm{~m}$ \\
\hline$I_{x}$ & 0.01896 & $\mathrm{~kg} \cdot \mathrm{m}^{2}$ \\
\hline$I_{y}$ & 0.01896 & $\mathrm{~kg} \cdot \mathrm{m}^{2}$ \\
\hline$I_{z}$ & 0.00939 & $\mathrm{~kg} \cdot \mathrm{m}^{2}$ \\
\hline
\end{tabular}

Table 2: Improved PSO Initialization Parameter

\begin{tabular}{|c|c|c|}
\hline Parameters & Values & Parameters \\
\hline$\omega_{\max }$ & 0.9 & $\omega_{\max }$ \\
\hline$\omega_{\min }$ & 0.4 & $\omega_{\min }$ \\
\hline$c_{1}, c_{1}$ & 2 & $c_{1}, c_{1}$ \\
\hline Particle swarm size & 20 & Particle swarm size \\
\hline Number of iterations & 50 & Number of iterations \\
\hline$h$ & 10 & $h$ \\
\hline
\end{tabular}

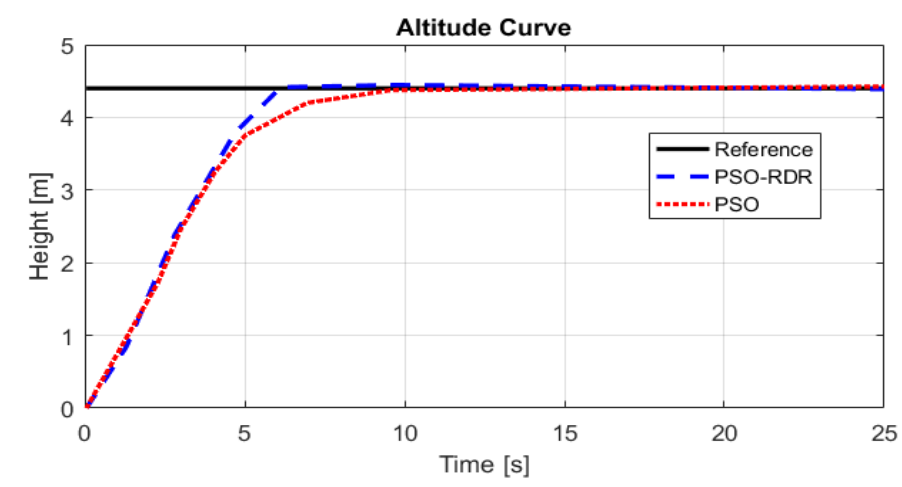

Figure: 2 Height control curve of UAV

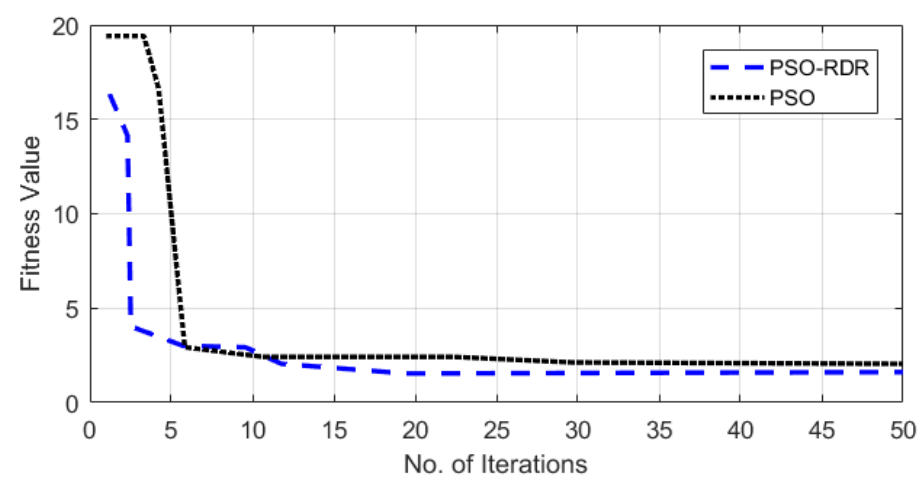

Figure: 3 No. of iterations for optimization

\section{Conclusion}

To conclude this article, it is observed that the designed PSO-RDR controller have better convergence speed for maneuvering control the altitude of UAV. To prove the efficiency of the proposed controller the results of both the standard PSO controller and robust disturbance rejection based PSO are compared in Simulink simulations, which 
shows the effectiveness of the designed controller. It can achieve precise and fast control of the height and attitude of the aircraft to improve the efficiency of flight control designed system.

\section{References}

[1] Emad Ebeid, Martin Skriver, Jie Jin. A Survey on Open-Source Flight Control Platforms of Unmanned Aerial Vehicle[C]// 2017 Euromicro Conference on Digital System Design (DSD). IEEE Computer Society, 2017.

[2] Fabian N. Murrieta-Rico, Daniel Hernandez-Balbuena, Julio C. Rodriguez-Quinonez. Resolution improvement of accelerometers measurement for drones in agricultural applications[C]// IECON 2016 - 42nd Annual Conference of the IEEE Industrial Electronics Society. IEEE, 2016.

[3] Ezat M A, Fritsch C J, Downs C T. Use of an unmanned aerial vehicle (drone) to survey Nile crocodile populations: A case study at Lake Nyamithi, Ndumo game reserve, South Africa [J]. 2018, 223:76-81.

[4] Weiyong Si, Haoping She, Zhanglong Wang. Fuzzy PID controller for UAV tracking moving target[C]// 2017 29th Chinese Control and Decision Conference (CCDC). IEEE, 2017.

[5] Xiaoqiong, L., Minghuan, L., Enzeng, D., \& Xiaoqiong, L. (2012). An adaptive GPC algorithm for a vectored water-jet-based spherical underwater vehicle.

[6] Jeng, Jyh-Cheng, Liao, Sinn-Jia. A Simultaneous Tuning Method for Cascade Control Systems Based on Direct Use of Plant Data [J]. Industrial \& Engineering Chemistry Research, 52(47):1682016831.

[7] Yuanhui Wang, Yunlong Yang, Fuguang Ding. Improved ADRC control strategy in FPSO dynamic positioning control application[C] 2016 IEEE International Conference on Mechatronics and Automation. IEEE, 2016.

[8] P. K. Kwok, D. W. H. Chau, H. Y. K. Lau. A Study on Road Junction Control Method Selection Using an Artificial Intelligent Multi-criteria Decision Making Framework [M]. Springer International Publishing, 2014.

[9] Mohd Shahrieel Mohd Aras, Shahrum Shah Abdullah, Hazriq Izuan Jaafar, Optimization of Single Input Fuzzy Logic Controller Using PSO for Unmanned Underwater Vehicle: Methods and Protocols [M]// CpG Islands. 2019.

[10] Remya Kommadath, Prakash Kotecha. Optimization of Stirling Engine Systems using Single Phase Multi-group Teaching Learning Based Optimization and Genetic Algorithm [M]// Smart Innovations in Communication and Computational Sciences. 2019.

[11] Laihong Zhou, Juqian Zhang, Houxin She, Quadrotor UAV flight control via a novel saturation integral backstepping controller [J]. Automatika, 2019, 60(2):193-206.

[12] Herman Castañeda, J. L. Gordillo. Spatial Modeling and Robust Flight Control Based on Adaptive Sliding Mode Approach for a Quadrotor MAV [J]. Journal of intelligent \& robotic systems: Theory \& applications, 2019, 93: págs. 101-111.

[13] Huayu Fan, Hao Zhan, Shixin Cheng, Research and Application of Multi-Objective Particle Swarm Optimization Algorithm Based on $\alpha$-Stable Distribution [J]. Xibei Gongye Daxue Xuebao/Journal of Northwestern Polytechnical University, 2019, 37(2):232-241. 
[14] Vippon Preet Kour, Sakshi Arora. Particle Swarm Optimization Based Support Vector Machine (PSVM) for the Segmentation and Classification of Plants [J]. IEEE Access, 2019.

[15] Rim Zarrouk, Imed Eddine Bennour, Abderrazek Jemai. A two-level particle swarm optimization algorithm for the flexible job shop scheduling problem [J]. Swarm Intelligence, 2019, 13(3). 